\title{
Geographical Routing in Intermittently Connected Ad Hoc Networks
}

\author{
Erik Kuiper \\ Linköping University \\ g-eriku@ida.liu.se
}

\author{
Simin Nadjm-Tehrani \\ Linköping University \\ snt@ida.liu.se
}

\begin{abstract}
In intermittently connected ad hoc networks standard routing protocols like AODV, DSR and GPSR fail since they generally cannot find $a$ contemporaneous path from source to destination. In this paper we present LAROD, a geographical routing protocol for intermittently connected networks. Combining beacon less geographical routing with store-carry-forward LAROD greedily searches for the shortest way to the destination and when no progress is possible packets are temporarily stored until node mobility has created a new path. In the paper we have shown by a comparative study that LAROD has almost as good delivery rate as an epidemic routing scheme, but at a substantially lower overhead.
\end{abstract}

\section{Introduction}

Ad hoc networking enables routing of messages in wireless networks between parties that cannot directly communicate with each other and where there is no fixed infrastructure. Mobile ad hoc networking can be useful in disaster scenarios or during military maneuvers. They could also be imagined in urban settings where you would like to create peer networks without relying on an (expensive) service provider.

Most routing algorithms for ad hoc networks require a contemporaneous path between sender and receiver. If no such path exists they will fail and the nodes cannot communicate. If this requirement is relaxed routing is possible in mobile networks by using a storecarry-forward model. A routing protocol will then use both wireless transfers and node mobility to transfer a message to its destination. An implication of the lack of a contemporaneous path is that the internet networking assumptions [1] are not met. The absence of an end-toend path means that long message delays will be the norm. A new generation of network routing protocols that address delay-tolerant networking have emerged to address this issue [16].
In this paper we present Location Aware Routing for Opportunistic Delay-Tolerant networks (LAROD). LAROD is a resource efficient geographical routing protocol for delay-tolerant networks (DTNs). When possible LAROD greedily forwards packets towards the destination and when no progress is possible a packet is temporarily stored by the current custodian. All packet forwarding is done without using beacons which means that system resources will not be used by nodes that do not take part in message forwarding.

LAROD uses a single-copy routing strategy which means that normally there only exists one copy of a packet in the network. Limiting the route search to a single route means that the network load is lower compared to multi-copy or epidemic schemes. This resource efficiency is attractive for resourceconstrained systems, but it is also beneficial for systems with a high network load.

LAROD has been evaluated in ns-2 [11] using a realistic scenario in which a group of unmanned aerial vehicles (UAVs) cooperate to perform reconnaissance of an area [7]. The evaluations have shown that LAROD has delivery rates close to the best achievable at a very low overhead.

In the next section we provide a background to routing in delay-tolerant networks. In section 3 we present LAROD and two other routing protocols used as a reference. In section 4 the routing protocols are evaluated and we then end the paper with some conclusions and ideas on future work.

\section{Background}

Routing in mobile ad hoc networks has been studied extensively and routing protocols like AODV [12], DSR [5] and GPSR [6] have been suggested. All these protocols assume that there exists a contemporaneous path between sender and receiver. In networks without contemporaneous paths, but where node mobility can overcome partitions, a new type of routing algorithm is required. 
In RFC 4838 [1] Cerf et al. describe an architecture for delay-tolerant and intermittently connected networks. Their architecture is designed for heterogeneous networks that are subject to long delays and/or discontinuous end-to-end connectivity. The architecture is based on asynchronous messaging and uses postal mail as a model of service classes and delivery semantics.

The mobility of the nodes does mean that the network topology will constantly change and that nodes constantly come in contact with new nodes and leave the communication range of others. In RFC 4838 Cerf et al. classify the contacts based on their predictability into scheduled, predicted and opportunistic contacts. With scheduled contacts the nodes know when they will be able to communicate with a specific peer. If nodes can estimate likely meeting times or meeting frequencies you have a network with predicted contacts. If no information is available on node contacts then the contacts are opportunistic. LAROD neither requires node contacts schedule nor predications and is thus well suited for networks with opportunistic contacts.

An overview of different routing strategies in delaytolerant networks can be found in Zhang's survey [17].

\subsection{DTN routing in opportunistic networks}

Routing in DTNs with opportunistic contacts is challenging since contact times and durations are not known in advance. Three examples of location unaware routing protocols for this environment are Randomized Routing [13], Epidemic Routing [15] and Spray and Wait [14]. In Randomized Routing only a single copy of a packet is present in the network. When two nodes meet a packet is handed over to the other at some set probability. This means that a packet randomly walks around in the network until it reaches the destination. This routing principle is better than keeping a packet at the source node until it comes in contact with the destination provided that the transmission speed is faster than the mode movement or if node movements are local.

In Epidemic Routing packets are distributed to all nodes in the network (or at least a considerably large subset of nodes) giving a high cost in both transfer and storage overhead. In Spray and Wait a packet is distributed to a small number of nodes. The nodes receiving the packet store it and the first node to meet the destination will deliver the packet. Spray and Wait can be an efficient protocol if the nodes that carry the packet cover a large part of the network with their mobility.
If the nodes are location aware and the (approximate) location of the destination is known then one can perform geographic routing. Li et al. [9] have modified GPSR [6] to better handle short temporary disruptions due to obstructions, node mobility or interference in relatively sparse networks (55 nodes $/ \mathrm{km}^{2}$ compared to our even more sparse scenario that has $10-30$ nodes $/ \mathrm{km}^{2}$ ). By using temporary storage (up to 2 seconds) and having a set of possibly reachable neighbors they substantially increased the delivery ratio compared to GPSR. LeBrun et al. [8] have performed geographical routing in a very sparse (0.3-4.4 nodes $\left./ \mathrm{km}^{2}\right)$ delay tolerant network with a stationary destination. Using a request-response mechanism beaconing is limited to the nodes that have data to transmit.

\subsection{Beacons-less routing}

Most routing protocols require knowledge of the neighbors of a node to make their routing decisions. This information is generally gathered by the use of beacons, messages broadcasted regularly that will be heard by all nodes within communication distance. A problem with beacons is that the gathered neighbor information is always to some extent old. Another issue is that beacons consume bandwidth, bandwidth that could be used for data transmissions. A problem for energy-constrained networks is that beacons consume energy irrespective of whether there is data to transmit or not. In [3] Heissenbüttel et al. study the impact of incorrect neighbor information due to beaconing for position based routing protocols.

An example of a beacon-less routing protocol is BLR by Heissenbüttel et al. [2]. BLR is a geographic routing protocol where nodes broadcast a packet when they want to send it. Nodes within a defined forwarding area are eligible to forward the packet and the actual node to forward a packet is determined by a timer function. For the best node the timer will expire first and it will then broadcast the packet. The other nodes waiting to forward the packet will hear this transmission and cancel their transmissions. If the greedy forwarding fails then a backup mode is entered and the packet is forwarded along the edge of a planar subgraph until the packet comes closer to the destination than it was when the backup mode was entered. To conserve energy and bandwidth BLR uses unicast routing for a period of time after a route has been established. To be able to handle position error and mobility of the destination a reactive local routing algorithm is used in the vicinity of the destination. 


\section{Proposed routing algorithms}

In this section we present LAROD and two other routing algorithms to be used in a comparative study.

\subsection{LAROD}

LAROD is a geographical routing protocol for DTNs that combines geographical beacon less routing with the store-carry-forward principle. In its essence LAROD uses greedy packet forwarding when possible. When greedy forwarding is not possible the node holding the packet (the custodian) waits until node mobility makes it possible to resume greedy forwarding.

When a node want to send a packet it simply broadcasts it. The best forwarding node receiving the message then forwards the message in the same manner. The node that sent the message will overhear this transmission and conclude that the forwarder has taken over custody of the packet. If no such transmission is heard the node regularly broadcast the message until a forwarder becomes available due to node mobility.

The nodes that are eligible to forward a packet are determined by a forwarding area related to the node performing the broadcast. In [2] Heissenbüttel et al. recommended a circle as the best forwarding area (see Figure 1a). Our simulations have shown that this area is too small due to the low density of nodes we use. Instead all nodes that provide some minimum progress towards the destination are eligible as forwarders (see Figure 1b). An implication of using this larger forwarding area is that multiple copies of a packet can be active since all nodes in the forwarding area might not hear the broadcast made by the best custodian. This should not be seen as a problem, since it enables exploration of multiple paths to the destination.

All nodes receiving the packet in the forwarding area are tentative forwarders and to select the next forwarder they all set a timer when to rebroadcast the packet. The timer function is a linear function with the highest delay at the sender and zero delay after some given point (as shown by the dashed lines in Figure 1). This prioritizes long steps minimizing the number of transmissions needed to reach the destination. To prevent simultaneous transmissions all nodes add a small random delay to the linear timer function.

The node with the timer that expires first will take custody of the packet and rebroadcast it. This broadcast will be heard by the old custodian and most of the other tentative forwarders. Overhearing the transmission they will remove their copy of the packet.

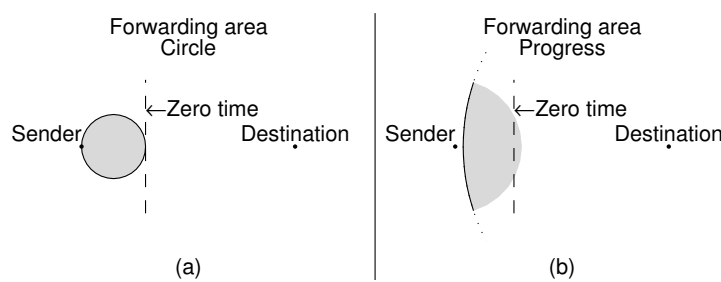

Figure 1. LAROD forwarding areas.

To stop transmission when a packet has reached its destination the destination sends an acknowledgement packet. To prevent that a packet indefinitely tries to find a path to its destination all packets have a time to live (TTL) expressed as a duration. When the TTL expires all nodes holding the packet removes it. For a full description of the routing protocol see Figure 2.

LAROD is based on the principle that the custody of a packet is moved to the most recent node that accepted the packet. This means that the protocol is not robust to node loss. If a node fails for some reason then all packets that the node holds will be lost unless they happened to be duplicated due to diverging paths.

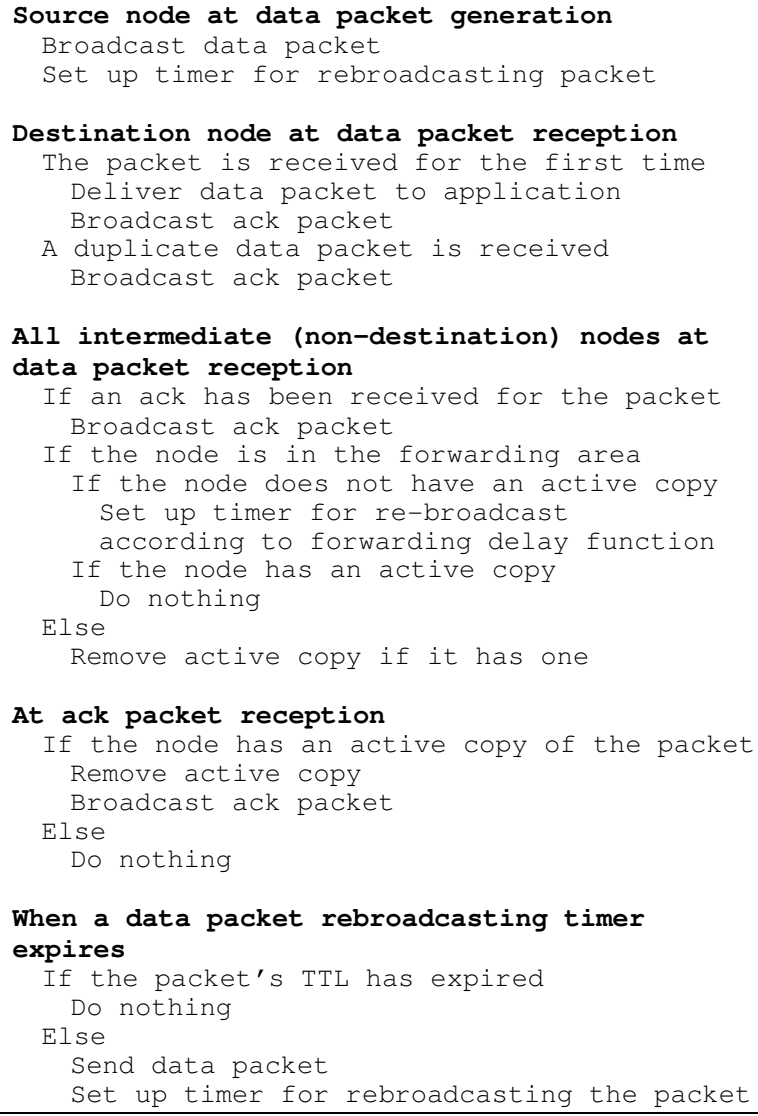

Figure 2. LAROD pseudo code 


\subsection{Broadcast Delay-tolerant Routing (BDTR)}

To be able to estimate the delivery rate of a delaytolerant routing protocol which searches all possible paths to a destination BDTR has been used. BDTR is a flooding based routing protocol that limits the flooding to an area centered round the source and destination. When a packet is received by a node for the first time it is rebroadcasted by the node after a short random delay if it is in the broadcast area. If there is a path to the destination in the broadcast area the packet will soon reach the destination. When the packet reaches the destination an acknowledgment packet is sent and it is in turn rebroadcasted by nodes that have received the data packet. To overcome partitions all nodes in the broadcast area regularly resend the data packet until an acknowledgement is received or the packet times out. For the simulations the broadcast area used was a circle with a diameter slightly larger than the distance between source and destination. This area means that not all possible paths are searched, but it gives a good balance between search space size and limiting the overhead. Tests with the complete simulation area as broadcast area have shown that the overhead was prohibitive and that it affected the ability to search all paths even at the low loads used in our simulations.

BDTR is a simple protocol and robust in the sense that several nodes have an individual packet and try to forward it to the destination. This means that failure of a single node does not impact the routing to a great degree. The rebroadcast and resend strategies used by BDTR means that it will quickly saturate the wireless medium in dense networks or networks that transmit large amounts of data. This means that BDTR by design in not suited for these environments.

\subsection{Broadcast Routing (BR)}

To evaluate how often a contemporaneous path exists between a source-destination pair a simple broadcast routing scheme was used. BR is identical to BDTR except that the nodes do not resend packets, they are only rebroadcasted once. For BR we have used the entire simulation area as broadcast area.

Table 1. Scenario parameters

\begin{tabular}{|l|l|}
\hline Reconnaissance area & 2000x2000 meters \\
\hline Placement of ground units & Center of each quadrant \\
\hline Node density & $10-30(20)$ nodes $/ \mathrm{km}^{2}$ \\
\hline UAV speed & $0.8-2.0(1.4) \mathrm{m} / \mathrm{s}$ \\
\hline Detect event life time & 400 seconds \\
\hline Detect data generation rate & $12-120(36) \mathrm{pkt} / \mathrm{hour} / \mathrm{UAV}$ \\
\hline Radio range & 250 meters \\
\hline
\end{tabular}

\section{Evaluation}

The above routing protocols have been implemented in a modified version of ns2 2.30 [11]. The main changes to the simulator are:

(1) addition of the main algorithm LAROD, and the new baselines (BDTR and its instance BR),

(2) addition of the pheromone repel mobility model [7],

(3) support for application level broadcasts,

(4) an application to generate and consume events.

As wireless communication technology we use IEEE 802.11 as implemented in ns2 2.30 with default parameters using the two-ray ground radio propagation model.

\subsection{Scenario}

The choice of scenario and scenario parameters are important since they will affect the result of the simulations [4][18]. This means that the scenario should be selected carefully to represent the environment in which the routing protocol is intended to be used.

In this paper we have used the reconnaissance scan scenario with the distributed pheromone repel mobility model presented in [7]. In the scenario a group of UAVs cooperate to perform reconnaissance of an area. To coordinate their scanning the UAVs exchange information with neighboring UAVs on recently visited areas. This information is stored in a pheromone map. Regularly a UAV evaluates where to go next by evaluating the pheromone map over the area in front of it. The selection where to go is done randomly but with higher probabilities given to directions leading to a not recently visited area. Compared to [7] all distances and speeds have been scaled down since we use 802.11 as the physical medium in our simulations.

When performing this scanning a UAV may detect something that needs to be communicated to a ground unit that can act on it. In the current simulations we have had four stationary ground units. The generation of detect events and selection of receiver are both randomly generated. In an ongoing extension of this work a publish subscribe technique is used for determining which ground unit(s) should get the relevant data packet. The main scenario parameters are presented in Table 1 where the values in parentheses are the default values.

Initially the UAVs are randomly distributed over the reconnaissance area. The simulation is first run for 1 hour without detect event packets being generated to populate the pheromone repel model with data. The detect events are then enabled for 1 hour and the 
simulation ends after all detect event packets have timed out. In the evaluations below each data point is the average result of ten simulations.

The node speed used in our simulations might seem low compared to the node speeds in other MANET papers [9][6], but it is actually close to the average speed used in most simulations. The speed presented in most papers is the top speed for the random waypoint mobility model. According to the equations in [10] for a top speed of $20 \mathrm{~m} / \mathrm{s}$ and minimum speed of $0.1 \mathrm{~m} / \mathrm{s}$ with no pause time $49.8 \%$ of the nodes would move slower than $1.4 \mathrm{~m} / \mathrm{s}$. Adding the fact that most simulations use pause times this means that they have large number of relatively stable nodes that can be used to create stable routes.

\subsection{Node Density}

The higher the node density the more connected the network will be and the easier it is to find a path between two nodes. As can be seen from the delivery ratio of BR in Figure 3 the node density has a dramatic impact on the existence of contemporaneous paths between source and destination. By using store-carryforward both LAROD and BDTR can overcome communication gaps and they significantly improve the delivery ratio compared to BR.

Even if LAROD and BDTR give about the same delivery ratio the overhead of BDTR is much higher as can be seen in the lower graph in Figure 3. The graph shows the average number of packets transmitted per data packet generated, that is the effort spent per data packet. The shape of the BDTR overhead curve is determined by two main factors. At low node densities only a few nodes receive a packet since fewer nodes are in the broadcast area. Additionally the clusters of connected nodes (partitions) will contain fewer nodes. Both these factors result in fewer transmissions generated at low node densities. As the node density increases more nodes will receive a packet and the number of transmissions increases. The reason the transmissions fall at higher node densities is that packets will be delivered faster to the destination due to increased connectivity. This means that the packet will live a shorter time in the network, reducing the number of transmissions.

\subsection{Node Speed}

A higher node speed should give a higher delivery ratio since the increased mobility will change the network topology at a higher pace enabling messages to bridge communication gaps faster. In Figure 4 we see that this is the case for both LAROD and BDTR. It is interesting to note that the delivery ratio for $\mathrm{BR}$ increases somewhat with higher mobility. This probably comes from the fact that BR has a short random delay between message reception and the resending of a packet. During this time the nodes will move making it possible to reach nodes that were not reachable at packet reception.

In Figure 4 we also see that increased node speed decreases the overhead for BDTR and LAROD. The cause is the improved delivery ratio at higher node speeds. Since most packets will be delivered before their expiry time fewer packets will be active in the network at higher delivery rates.

\subsection{Network load}

To investigate the scalability of the routing protocols when it comes to network load different event generation rates were tested. Ideally a fully scalable protocol will give the same result independent of network load. In Figure 5 we see that the delivery ratio of BDTR drops as the load increases while it stays constant for LAROD. The problem for BDTR is that the network becomes congested and that several transmissions fail due to collisions. This clearly shows that the broadcast strategy of BDTR has scalability issues. For LAROD the critical load level is not yet reached and as a result the delivery ratio is stable. In Figure 5 we also see the impact of the lower delivery ratio on the load for BDTR. As the delivery ratio decreases the packets live longer in the network and as a result the per packet overhead increases.

\section{Conclusions and future work}

In the paper we have shown that geographical routing in intermittently connected networks using a store-carry-forward technique gives almost as good delivery rate as a store-carry-forward broadcast routing scheme, but at a substantially lower overhead. This lower overhead means that node resources like power and storage will be taxed lower, making a higher network utilization possible. We have also shown that routing protocols that rely on the existence of a contemporaneous path will have delivery rates in intermittently connected networks that make them practically unusable in these environments.

The Achilles heel of geographical routing is how the position of the destination is made known to the source. We plan to investigate how a location service could be designed to work in a DTN environment where distribution of information takes much time. 

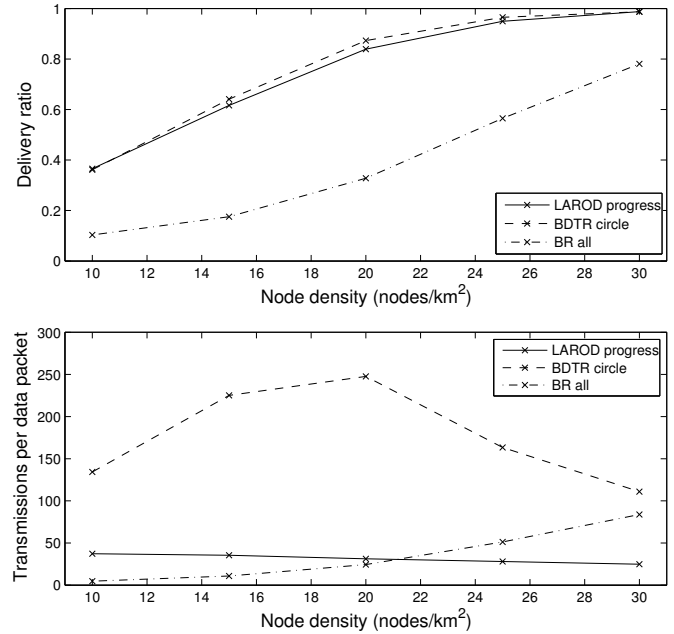

Figure 3. Node density results.
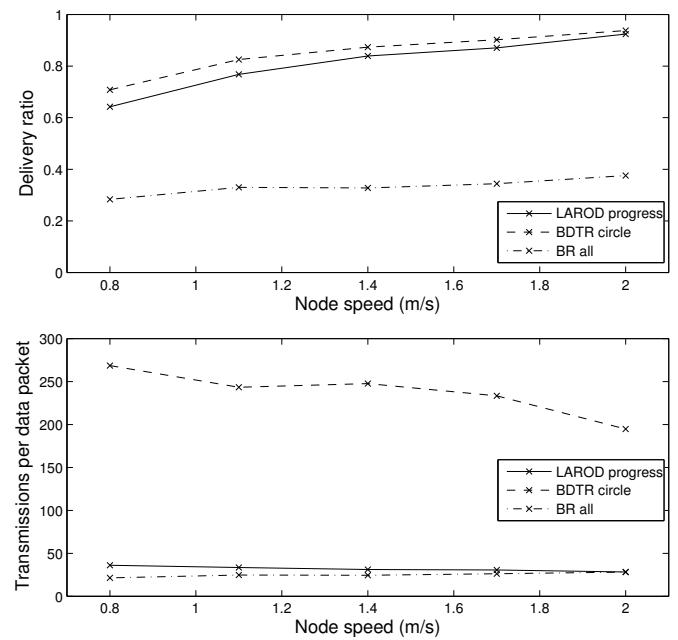

Figure 4. Node speed results.
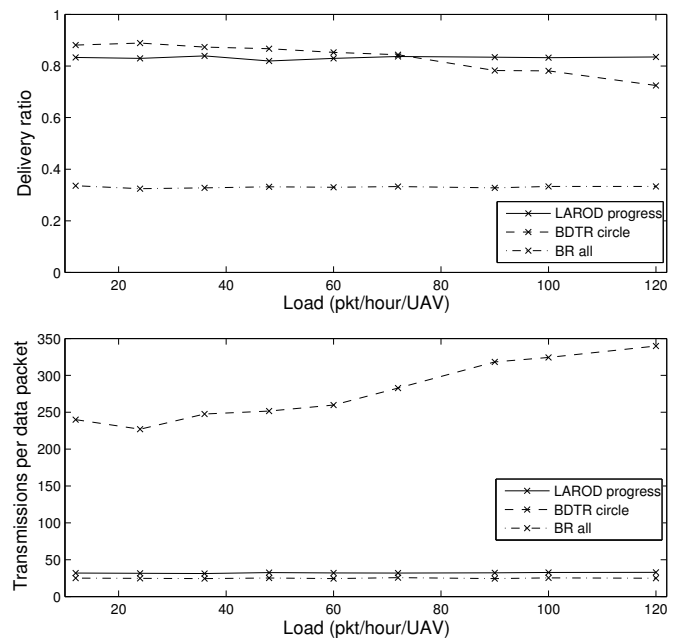

Figure 5. Network load results.

\section{References}

[1] V. Cerf, S. Burleigh, A. Hooke, L. Torgerson, R. Durst, K. Scott, K. Fall, H. Weiss. Delay-tolerant networking architecture. RFC 4838. April 2007. RFC Editor (ftp://ftp.rfc-editor.org/in-notes/rfc4838.txt)

[2] M. Heissenbüttel, T. Braun, T. Bernoulli, M. Wälchi. BLR: beacon-less routing algorithm for mobile ad hoc networks. Computer communications. July 2004. Elsevier [3] M. Heissenbüttel, T. Braun, M. Wälchi, T. Bernoulli. Evaluating the Limitations of and Alternatives in Beaconing. Ad Hoc Networks. Vol. 5 Iss. 5, July 2007. Elsevier

[4] A. Jardosh, E. M. Belding-Royer, K. C. Almeroth, S. Suri. Towards Realistic Mobility Models for Mobile Ad Hoc Networks. 9th annual International Conference on Mobile Computing and Networking. September 2003. ACM

[5] D. Johnson, D.A. Maltz. Dynamic Source Routing in Ad Hoc Wireless Networks. Mobile Computing, 1996. Kluwer

[6] B. Karp, H.T. Kung. GPSR: Greedy perimeter stateless routing for wireless networks. 6th annual ACM/IEEE International Conference on Mobile Computing and Networking (MobiCom). 2000. ACM/IEEE

[7] E. Kuiper, S. Nadjm-Tehrani. Mobility Models for UAV Group Reconnaissance Applications. ICWMC. 2006. IEEE

[8] J. LeBrun, C. Chuah, D. Ghosal, M. Zhang. KnowledgeBased Opportunistic Forwarding in Vehicular Wireless Ad Hoc Networks. VTC-Spring. 2005. IEEE

[9] Y. Li, T.H. Lai, M.T. Liu, M. Sun, J. Yang. DTGR: Disruption-tolerant geographic routing for wireless ad hoc networks. Simulation. June 2006. Sage

[10] W. Navidi, T. Camp. Stationary Distributions for the Random Waypoint Mobility Model. IEEE Transactions on Mobile Computing. Jan 2004. IEEE

[11] http://nsnam.isi.edu/nsnam/index.php/Main_Page [12] C.E. Perkins, E.M. Royer. Ad-hoc On-Demand Distance Vector Routing. WMCSA. 1999. IEEE

[13] T. Spyropoulos, K. Psounis, C.S. Raghavendra. Singlecopy routing in intermittently connected mobile networks. Sensor and Ad Hoc Communications and Networks. 2004 IEEE

[14] T. Spyropoulos, K. Psounis, C.S. Raghavendra. Spray and wait: an efficient routing scheme for intermittently connected mobile networks. SIGCOMM, WDTN. 2005. $\mathrm{ACM}$

[15] A. Vahdat, D. Becker. Epidemic Routing for PartiallyConnected Ad Hoc Networks. Tech. Rep. CS-2000-06, Duke University. 2000

[16] F Warthman. Delay-Tolerant Networks (DTNs): A Tutorial v1.1. March 2003. Available at http://www.dtnrg.org/wiki/Docs. Accessed October 15, 2007

[17] C. Zhang. Routing in Intermittently Connected Mobile Ad Hoc Networks and Delay tolerant networks: Overview and Challenges. IEEE Communication Surveys. Jan 2006. IEEE

[18] B. Zhou, K. Xu, M. Gerla. Group and Swarm Mobility Models for Ad Hoc Network Scenarios Using Virtual Tracks. Military Communication Conference. 2004. IEEE 